\title{
Direct Measurement of the Breakup Process
}

\author{
F. A. Souza, R. Liguori Neto, M. M. de Moura, M. G. Munhoz, A. A. P. \\ Suaide, E. M. Szanto, J. Takahashi, A. Szanto de Toledo, and N. Carlin \\ Instituto de Física - Universidade de São Paulo, Departamento de Física Nuclear, \\ Laboratório Pelletron, Caixa Postal 66318, 05315-970, São Paulo - SP, Brazil
}

Received on 15 November, 2004

\begin{abstract}
In order to better understand the breakup contribution to fusion, we perfomed light particle coincidence measurements for the systems ${ }^{6,7} \mathrm{Li}+{ }^{12} \mathrm{C},{ }^{59} \mathrm{Co}$ at energies near and above the barrier. A three body kinematics analysis is performed with the objective of separating the contributions of different reaction mechanisms. For small angular differences between the light particles, the sequential and direct breakup seem to dominate. On the other hand, for large detector separations, a sequential decay following transfer is likely to dominate.
\end{abstract}

The recent availability of radioactive nuclei beams motivated the investigation of fusion reactions involving weakly bound nuclei. The cross section enhancement generally observed at sub-barrier energies is understood in terms of dynamical processes arising from couplings to collective inelastic excitations of the target and/or projectile [1]. However, in the case of reactions where at least one of the colliding nuclei has a sufficient low binding energy so that breakup becomes an important process, conflicting experimental and theoretical results are reported in recent papers [2-10].

The ${ }^{6,7} \mathrm{Li}+{ }^{12} \mathrm{C},{ }^{59} \mathrm{Co}$ fusion reactions are used to investigate the effect of breakup on the fusion cross section [11]. These measurements help to establish the influence of the projectile breakup on the fusion process at near-barrier energies and contribute to the determination of how the mass of the target affects the process, as well as the influence of the incomplete fusion yield.

It is important to have clear the reference used when an enhancement and/or a suppression is defined[5-7]. A final tuning for the coupling of the breakup channel, as well as the correct description of the reaction dynamics, requires the explicit measurement of precise elastic scattering data as well as yields leading to breakup itself [12-14].
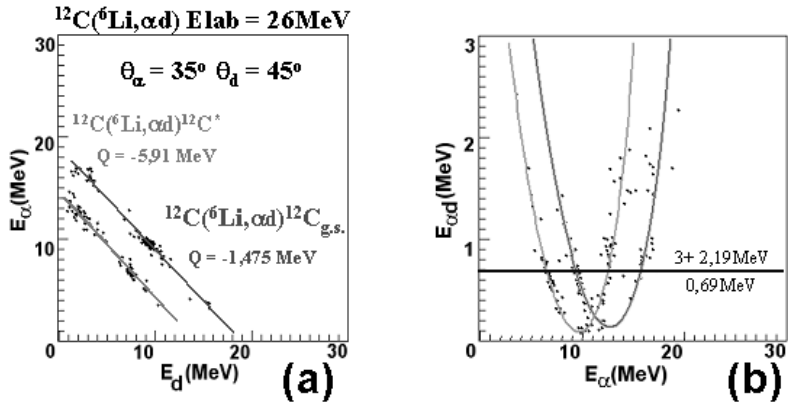

FIG. 1: (a) Bidimensional plot $E_{\alpha} \times E_{d}$. (b) Bidimensional plot $E_{\alpha d} \mathrm{x} E_{\alpha}$, both at $E_{\text {lab }}=26 \mathrm{MeV}$ for the ${ }^{6} \mathrm{Li}+{ }^{12} \mathrm{C}$ system. The lines represents the loci for events leaving ${ }^{12} \mathrm{C}$ in the ground state and the first excited state.

The identification of the breakup products has been achieved measuring the three body final state correlations. Coincidence data displayed in Fig. 1a show the identification of

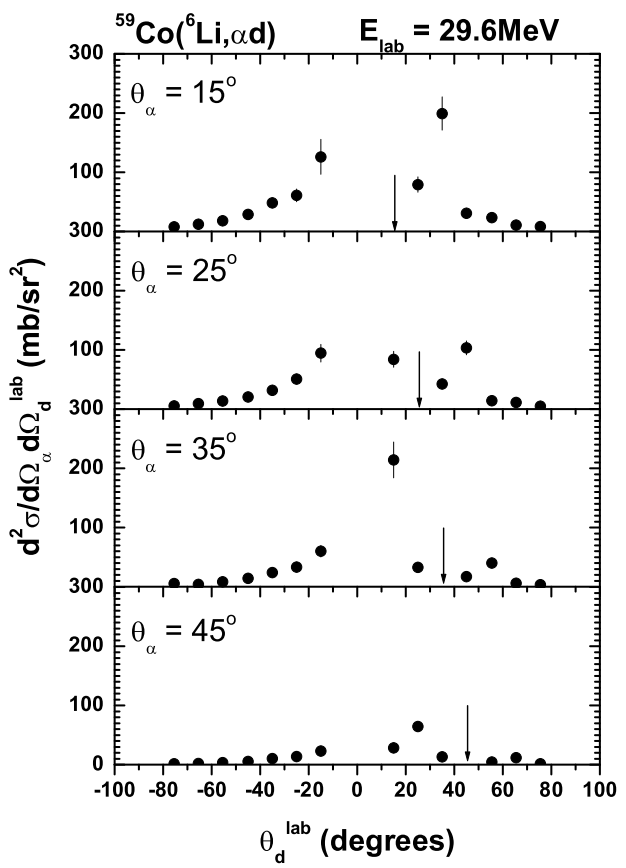

FIG. 2: Angular correlations for the system ${ }^{6} \mathrm{Li}+{ }^{59} \mathrm{Co}$ at $E_{\text {lab }}=$ $29.6 \mathrm{MeV}$ as a function of the $d$ angle $\left(\theta_{d}\right)$ for fixed $\alpha$ angles $\left(\theta_{\alpha}\right)$ indicated by the arrows.

the process Q-value in order to gate exclusively on the projectile breakup channel. Furthermore, the system excitation energy as well as the projectile fragment relative energy (see Fig. 1b) are used to identify the exit channel with no ambiguity. Based on those filters, angular correlations (see Fig. 2) are obtained to identify the several processes. This is complemented by measurements of relative energy of the fragments using different rest frame references (target like, projectile, target + fragment) in order to disentangle the contribution of breakup, incomplete fusion and/or transfer-reemission process. These experimental results are compared to three body kinematics calculations. As an example Fig. 3 displays the relative energy between $\alpha-d$ for the ${ }^{6} \mathrm{Li}$ breakup on a ${ }^{12} \mathrm{C}$ 


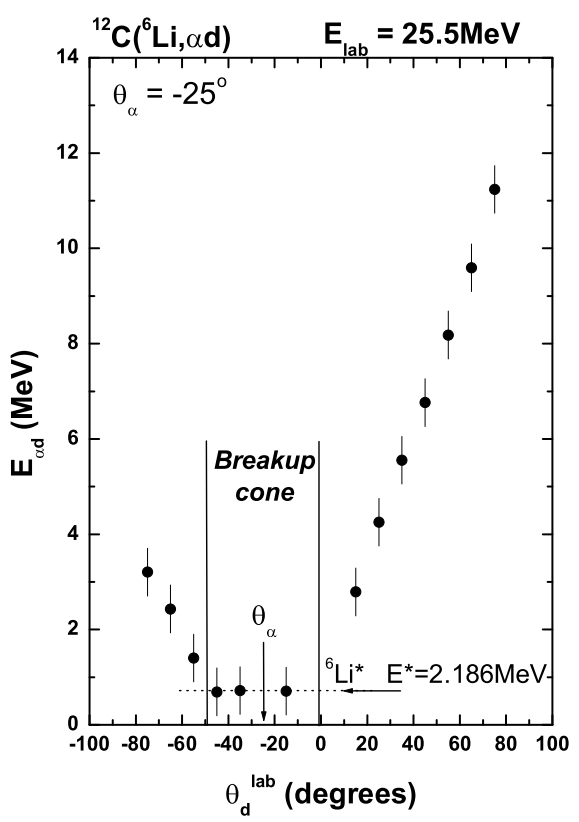

FIG. 3: Relative energy between $\alpha$ and $d$ for the ${ }^{6} \mathrm{Li}$ breakup on the ${ }^{12} \mathrm{C}$ target as a function of $\theta_{d}$ for a fixed $\theta_{\alpha}=-25^{\circ}$. The vertical lines represent the kinematical detection limits for $\alpha-d$ decay for the first excited state of ${ }^{6} \mathrm{Li}\left(\mathrm{E}^{*}=2.186 \mathrm{MeV}\right)$.

target. The region between the dashed vertical lines represent the angular interval in which the three body kinematics predict a constant relative energy. The fact that this experimental trajectory is not constant out of this region indicates the presence of other processes. The fact that the yield for coincidences is not negligible out of this region (see Figs. 2) indicates that the other processes producing, as in this case $\alpha-d$ coincidences, are relevant and must be identified. Fig. 4 shows the $\alpha$ energy as a function of the $d$ emission angle. We notice that the $\alpha$ energy is constant on the angular range which does not correspond to the breakup contribution. This constant energy suggests the presence of a binary intermediate process ${ }^{6,7} \mathrm{Li}+{ }^{59} \mathrm{Co} \rightarrow \alpha+{ }^{61} \mathrm{Ni}^{*} \rightarrow \alpha+d+{ }^{59} \mathrm{Co}$. From the average $\alpha$ energy, we determined the excitation energy of the intermediate nucleus ${ }^{61} \mathrm{Ni}$, as being $25 \mathrm{MeV}$. Similar data were taken for ${ }^{6,7} \mathrm{Li}+{ }^{115} \mathrm{In}$. This procedure in unfolding the several light particle emission processes has not been exploited so far in the literature.

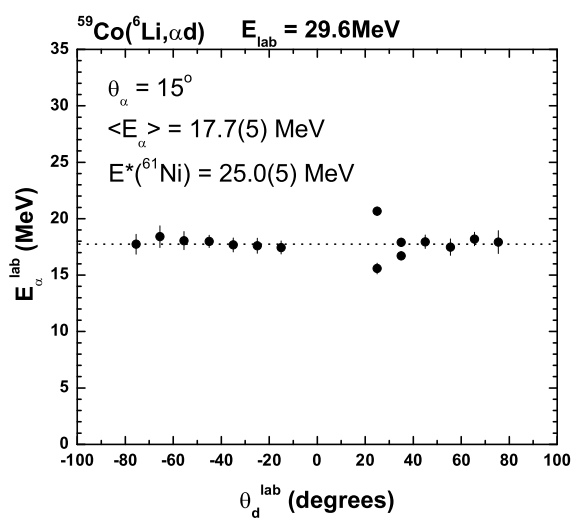

FIG.4: $\alpha$ energy as a function of $\theta_{d}$ for $\theta_{\alpha}=15^{\circ}$. The excitation energy for the intermediate nucleus ${ }^{61} \mathrm{Ni}$ was determined from the average $\alpha$ energy indicated by the dotted line.

\section{Acknowledgments}

This work was supported by Fundação de Amparo à Pesquisa do Estado de São Paulo (FAPESP)-Brasil.
[1] M. Dasgupta et al., Ann. Rev. Nucl. Part. Sci. 48, 401 (1998) and references therein.

[2] N. Takigawa et al., Phys. Rev. C 47, R2470 (1993).

[3] K. Hagino et al., Phys. Rev. C 61, 037602 (2000) and references therein.

[4] A. Diaz-Torres and I.J. Thompson, Phys. Rev. C 65, 024606 (2002).

[5] J.Takahashi et al., Phys. Rev. Lett. 78, 30 (1997).

[6] A. Szanto de Toledo et al., Nucl. Phys. A 679, 175 (2000).

[7] R.Cabezas et al., Phys. Rev. C 60, 067602 (1999).
[8] M .Dasgupta et al., Phys. Rev. Lett 82, 1395 (1999).

[9] M .Dasgupta et al., Phys. Rev. C 66, 041602R (2002).

[10] V.Tripathi et al., Phys. Rev. Lett. 88, 172701-1 (2001).

[11] C. Beck et al., Phys. Rev. C 67, 54602 (2003).

[12] K. Rusek et al., Phys. Rev. C 67, 41604 (2003).

[13] J.F. Liang et al., Phys. Rev. C 67, 44603 (2003).

[14] C.Signorini et al., Phys. Rev. C 67, 44607 (2003) and references therein. 